\title{
Frequency Transmission Control of Local Networked Control Systems Approach
}

\author{
O. Esquivel-Flores*${ }^{* 1}$, H. Benítez-Pérez ${ }^{2}$ \\ ${ }^{1}$ Universidad Nacional Autónoma de México \\ Posgrado en Ciencia e Ingeniería de la Computación \\ *oaefmcc@hotmail.com \\ ${ }^{2}$ Universidad Nacional Autónoma de México \\ Departamento de Ingeniería de Sistemas Computacionales y Automatización \\ Instituto de Investigación en Matemáticas Aplicadas y en Sistemas
}

\begin{abstract}
The use of Network Control Systems has been successful in the industry and therefore has opened several lines of research. Control systems over a communication network include two important considerations, control and scheduling. Co-design strategies are focused on maintaining adequate control performance and maximize the level of service. Network scheduling is the main objective to resolve because through a balanced data load is possible to maintain control performance in a desired level. This article reviews a scheduling strategy based on frequency transition modeled as a linear subsystem, this control reconfigure on line the sensors' periods. The case of study is the control of a prototype helicopter and by numerical simulations it shows the effect of changing the transmission frequency.
\end{abstract}

Keywords: distributed systems, transmission frequency, control.

\section{RESUMEN}

Actualmente los sistemas de control en red han sido utilizados ampliamente en la industria y han tomado gran interés en el campo de la investigación. Dos áreas de estudio son consideradas en los sistemas de control en red: el control y la planificación. Las estrategias de co-diseño se enfocan en mantener un nivel de control y maximizar al mismo tiempo el nivel de servicio del medio de comunicación. La planificación del medio de red es el principal objetivo a resolver dado que al balancear la carga de los datos que se transmiten se mantiene el desempeño de control en un nivel deseado. Este artículo revisa una estrategia de planificación basado en el cambio de frecuencias modelado como un subsistema lineal, tal control reconfigura en línea los periodos de muestreo de los sensores del sistema. El caso de estudio es el control de un prototipo de helicóptero y por medio de simulaciones numéricas se muestra el efecto del cambio de frecuencias de transmisión de datos.

\section{Introduction}

The study of networked control systems (NCS) has increased considerably in recent years because these systems have high reliability, are low cost and easy to maintain. In a NCS, performance level lies not only in the controller design but also in scheduling the common communication media. Several considerations must be taken to design NCSs in which the control is not affected by the choice of high sampling rates at which networks with limited bandwidth leads to delays and losing data. A problem to be solved in the analysis and design of an NCS is to choose an accurate sampling period such that scheduling achieves the best performance of the system. A particular

feature of NCS is the presence of time delays since sharing common communication media. Several strategies have been proposed to ensure performance of control in the presence of delays [14]. Moreover, scheduling of an NCS has been widely studied [5-7], several algorithms have been proposed to achieve the highest level of resource utilization and minimize the effect of time delays. A strategy to obtain an appropriate quality service level and quality control level is the reconfiguration of states [15]. Co-design techniques have been implemented to balance control and scheduling; reconfigurable control is another strategy for handling time delays. Consider the reconfiguration 
as a transition that changes the structure of a system so that their representation of state changes, therefore by modifying the sampling periods of the nodes involved in an NCS is possible to lead the system into a schedulable state.

In order to choose an appropriate sampling rate, Lian et al. [5] provide a clear way to choose a sampling period to minimize the effect of delays. Authors define the relationship between control performance and sampling period establishing a performance degradation point, named period in digital control, and two points $b$ and $c$ corresponding to sampling periods $p_{b}$ and $p_{c}$ where the system lives in valid conditions within this range. Following this concept as a starting point, recently there have been proposals to find balance between sampling periods and the amount of data that can be transmitted through the network. Peng et al. [8] modify the transmission frequency to obtain optimum performance, frequency is the inverse of the period $(f=1 / p)$. From the beginning, they consider the system schedulable and place special emphasis on control performance and quality of service constraints to propose a linear programming problem whose solution is a proper data transmission rate.

This paper presents a first approximation to the operation of a scheduler that reconfigures the data transmission rate of the sensors in an NCS considering transmission frequencies as a subsystem that can be controlled into a bounded region by maximum and minimum transmission values where the system is schedulable. As a case of study, this proposal is implemented into flight control of a prototype helicopter. Numerical simulations using Matlab and Simulink are provided to show the proposal performance and overall system performance.

\section{Case of study and problem formulation}

The distributed system used for implementation purposes of this paper is a 2-DOF helicopter prototype [10]. The following sections briefly introduce and describe this prototype and its controller design, as well as an experimental approach to express this prototype as an NCS

\subsection{The 2-dof helicopter dynamics and control design}

The case study is a prototype of a helicopter system integrated to a CANBus (Controller Area Networks) network with two propellers that are driven by DC motors. The front propeller controls the elevation of the helicopter nose about the pitch axis and the back propeller controls the side-toside motions of the helicopter about the yaw axis. The pitch and yaw angles are measured using high-resolution encoders. In here, a brief description of the helicopter model is presented, however, detailed information can be found in [10]. The dynamics of the helicopter is developed based on kinetic and potential energy; this model is used to design a position controller. The center of mass of the helicopter is described in the $x y z$ cartesian coordinates with respect to pitch $\theta$, and yaw $\psi$, angles (Figure 1).

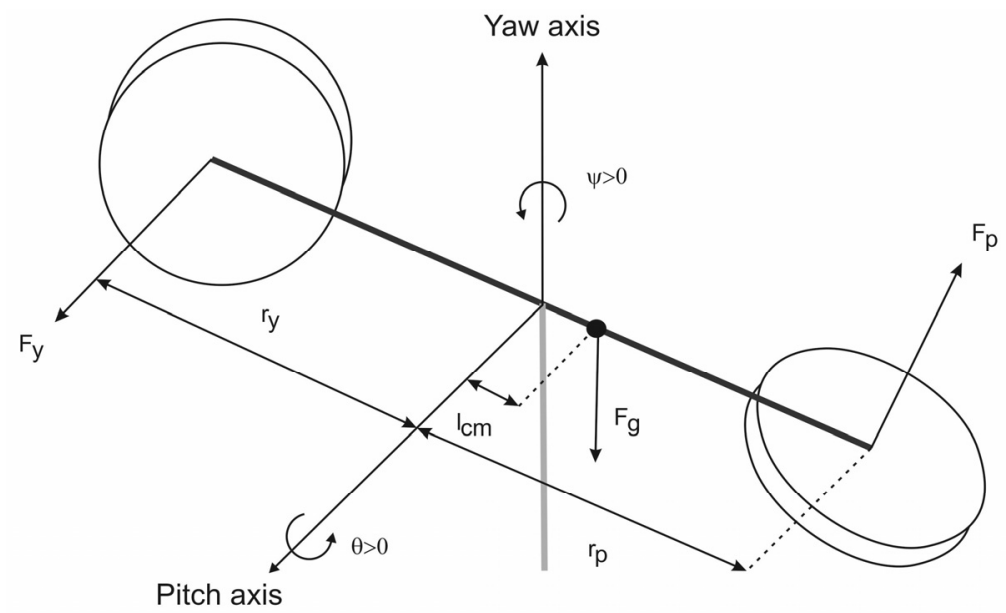

Figure 1. Helicopter Dynamics. 
Euler-Lagrange equations are used to obtain the nonlinear equation of motion for the 2 DOF Helicopter which will be used to derive the linear state model and, subsequently, to design the position controller. Table 1 shows specifications and parameters associated to the system.
A way to found nonlinear equations of motion is to use Euler-Lagrange equations. In this work this method is not reviewed. This Eulear-Lagrange method requires the generalized coordinate and forces which involve pitch and yaw derivatives $\dot{\theta}, \dot{\psi}$ Evaluating the Euler-Lagrange expressions and linearizing around the point:

\begin{tabular}{|c|c|c|c|c|}
\hline Symbol & $\begin{array}{l}\text { Matlab } \\
\text { Notation }\end{array}$ & Description & Value & Unit \\
\hline $\mathrm{B}_{\mathrm{cq}, \mathrm{p}}$ & B_eq_p & Equivalent viscous damping about pitch axis & 0.800 & N/V \\
\hline $\mathrm{B}_{\mathrm{cq}, \mathrm{y}}$ & B_eq_y & Equivalent viscous damping about yaw axis & 0.318 & $\mathrm{~N} / \mathrm{V}$ \\
\hline$m_{\text {heli }}$ & m_heli & Mass of the helicopter & 1.387 & $\mathrm{~kg}$ \\
\hline $\mathrm{m}_{\mathrm{m}, \mathrm{p}}$ & m_motor_p & Mass of pitch motor & 0.292 & $\mathrm{~kg}$ \\
\hline $\mathrm{m}_{\mathrm{m}, \mathrm{y}}$ & m_motor_y & Mass of yaw motor & 0.128 & $\mathrm{~kg}$ \\
\hline $\mathrm{m}_{\text {shield }}$ & m_shield & Mass of propeler shield & 0.167 & $\mathrm{~kg}$ \\
\hline $\mathrm{m}_{\text {props }}$ & m_props & $\begin{array}{l}\text { Mass of pitch and yaw propelers, propeler } \\
\text { shields and motors }\end{array}$ & 0.754 & $\mathrm{~kg}$ \\
\hline $\mathrm{m}_{\text {body }, \mathrm{p}}$ & m_body_p & Mass moving about the pitch axis & 0.633 & $\mathrm{~kg}$ \\
\hline $\mathrm{m}_{\text {body,y }}$ & m_body_y & Mass moving about the yaw axis & 0.667 & $\mathrm{~kg}$ \\
\hline$m_{\text {shaft }}$ & m_shaft & $\begin{array}{l}\text { Mass of the metal shaft rotating about the } \\
\text { yaw axis }\end{array}$ & 0.151 & $\mathrm{~kg}$ \\
\hline $\mathrm{L}_{\text {body }}$ & L_body & Total length of helicopter body & 0.483 & $\mathrm{~m}$ \\
\hline $\mathrm{I}_{\mathrm{cm}}$ & I_cm & $\begin{array}{l}\text { Center of mass length along helicopter body } \\
\text { from pitch axis }\end{array}$ & 0.186 & $\mathrm{~cm}$ \\
\hline $\mathrm{L}_{\text {shaft }}$ & L_shaft & $\begin{array}{l}\text { Length of metal shaft rotating about the yaw } \\
\text { axis }\end{array}$ & 0.280 & $\mathrm{~m}$ \\
\hline$J_{\text {body,p }}$ & J_m_p & $\begin{array}{l}\text { Moment of inertia of helicopter body about } \\
\text { the pitch axis }\end{array}$ & 0.012 & $\mathrm{~kg} \cdot \mathrm{m}^{2}$ \\
\hline $\mathrm{J}_{\text {body,y }}$ & J_m_y & $\begin{array}{l}\text { Moment of inertia of helicopter body about } \\
\text { the yaw axis }\end{array}$ & 0.013 & $\mathrm{~kg} \cdot \mathrm{m}^{2}$ \\
\hline $\mathrm{J}_{\text {shaft }}$ & J_shaft & $\begin{array}{l}\text { Moment of inertia of metal shaft about yaw } \\
\text { axis at end point }\end{array}$ & 0.004 & $\mathrm{~kg} \cdot \mathrm{m}^{2}$ \\
\hline$J_{p}$ & J_p & $\begin{array}{l}\text { Moment of inertia of front motor/shield } \\
\text { assembly about pitch pivot }\end{array}$ & 0.018 & $\mathrm{~kg} \cdot \mathrm{m}^{2}$ \\
\hline $\mathrm{J}_{\mathrm{y}}$ & J_y & $\begin{array}{l}\text { Moment of inertia of back motor/shield } \\
\text { assembly about yaw pivot }\end{array}$ & 0.008 & $\mathrm{~kg} \cdot \mathrm{m}^{2}$ \\
\hline$J_{\text {eq,p }}$ & J_eq_p & Total moment of inertia about pitch pivot. & 0.038 & $\mathrm{~kg} \cdot \mathrm{m}^{2}$ \\
\hline$J_{\text {eq,y }}$ & J_eq_y & Total moment of inertia about yaw pivot & 0.043 & $\mathrm{~kg} \cdot \mathrm{m}^{2}$ \\
\hline
\end{tabular}

Table 1. Helicopter Parameters. 


$$
\left(\theta_{0}=0, \Psi_{0}=0, \dot{\theta}_{0}=0, \dot{\psi}_{0}=0\right)
$$

thus,

$$
\begin{aligned}
& \left(\mathrm{J}_{\text {eq,p }}+\left.\mathrm{m}_{\text {heli }}\right|_{\mathrm{cm}} ^{2}\right) \ddot{\theta}=\mathrm{K}_{\mathrm{pp}} \mathrm{V}_{\mathrm{m}, \mathrm{p}}+\mathrm{K}_{\mathrm{py}} \mathrm{V}_{\mathrm{m}, \mathrm{y}}-\mathrm{B}_{\mathrm{p}} \dot{\theta}-\mathrm{m}_{\text {heli }} \mathrm{g} \mathrm{l}_{\mathrm{cm}} \\
& \left(\mathrm{J}_{\text {eq,y }}+\left.\mathrm{m}_{\text {heli }}\right|_{\mathrm{cm}} ^{2}\right) \ddot{\Psi}=\mathrm{K}_{\mathrm{yy}} \mathrm{V}_{\mathrm{m}, \mathrm{y}}+\mathrm{K}_{\mathrm{yp}} \mathrm{V}_{\mathrm{m}, \mathrm{p}}-\mathrm{B}_{\mathrm{y}} \dot{\Psi}-2 \mathrm{~m}_{\text {heli }} \mathrm{l}_{\mathrm{cm}}^{2} \theta \dot{\psi} \dot{\theta}
\end{aligned}
$$

Substituting $x=\left[\begin{array}{llll}\theta & \Psi & \dot{\theta} & \dot{\psi}\end{array}\right]^{\top}$ in (2) and (3) and solving for $\dot{x}$, we obtain a linear model of state spaces:

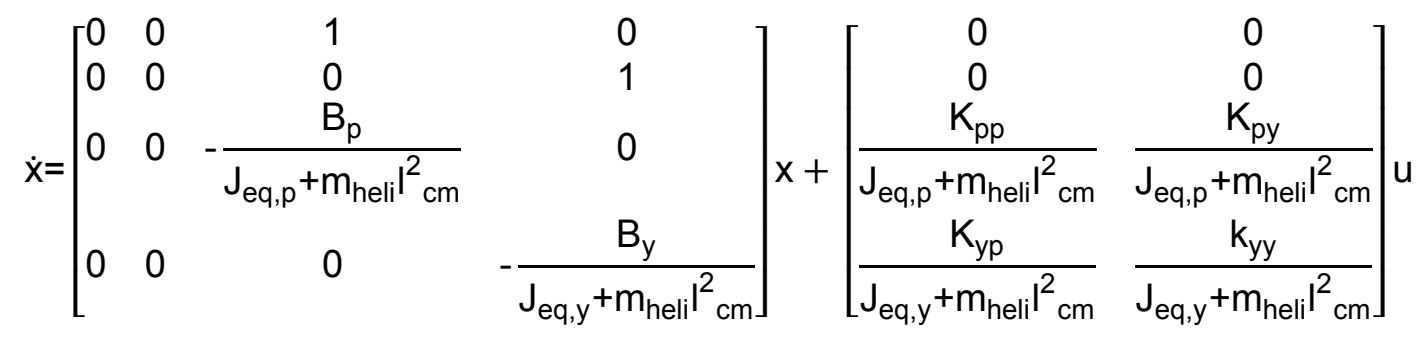

$$
y=\left[\begin{array}{llll}
1 & 0 & 0 & 0 \\
0 & 1 & 0 & 0 \\
0 & 0 & 1 & 0 \\
0 & 0 & 0 & 1
\end{array}\right] x
$$

The state of the model is the vector:

$$
x=\left[\begin{array}{llll}
\theta & \psi & \dot{\theta} & \dot{\psi}
\end{array}\right]^{\top}
$$

Input $u$ and output y are

$$
\begin{aligned}
& u=\left[\mathrm{V}_{\mathrm{m}, \mathrm{p}}, \mathrm{V}_{\mathrm{m}, \mathrm{y}}\right] \\
& \mathrm{y}=\left[\mathrm{x}_{1}, \mathrm{x}_{2}, \mathrm{x}_{3}, \mathrm{x}_{4}\right]^{\top}
\end{aligned}
$$

$\theta$ is the pitch angle, $\psi$ the yaw angle, $\dot{\theta}$ pitch derivative, $\dot{\psi}$ yaw derivative, $V_{m, p}$ is the input pitch motor voltage and $V_{m, y}$ is the input yaw motor voltage. All states are measurable.

The FF+LQR control that converges $(\theta, \psi, \dot{\theta}, \dot{\psi}) \rightarrow(\theta, \psi, 0,0)$ where $\theta_{d}$ is the desired pitch angle and $\Psi_{\mathrm{d}}$ is the desired yaw angle is

$$
\left[\begin{array}{l}
u_{p} \\
u_{y}
\end{array}\right]=\left[\begin{array}{c}
k_{\text {ff }} \frac{m_{\text {heligl } m \cos \theta_{d}}}{k_{p p}} \\
0
\end{array}\right]-\left[\begin{array}{llll}
k_{11} & k_{12} & k_{13} & k_{14} \\
k_{21} & k_{22} & k_{23} & k_{24}
\end{array}\right]\left[\begin{array}{c}
\theta-\theta_{d} \\
\Psi-\Psi_{d} \\
\dot{\theta} \\
\dot{\psi}
\end{array}\right]
$$




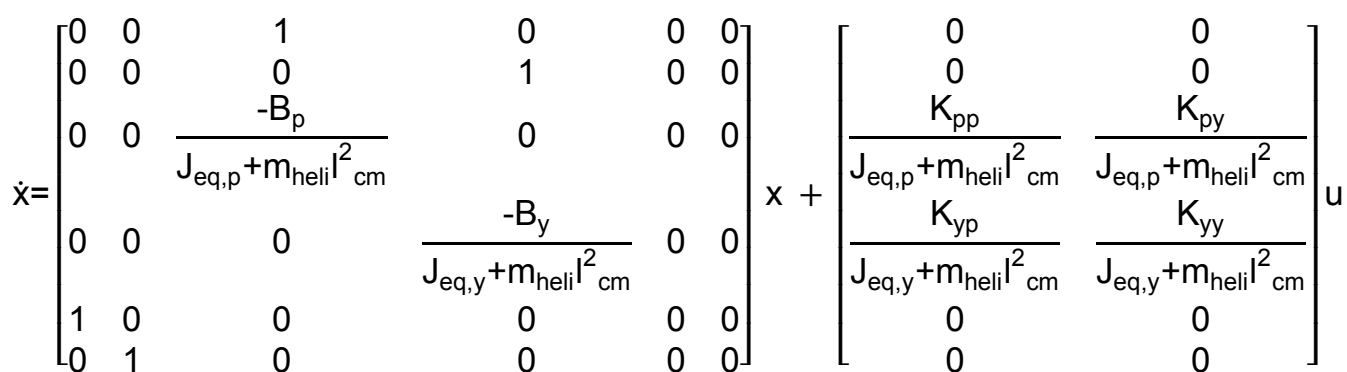

$y=\left[\begin{array}{llllll}1 & 0 & 0 & 0 & 0 & 0 \\ 0 & 1 & 0 & 0 & 0 & 0 \\ 0 & 0 & 1 & 0 & 0 & 0 \\ 0 & 0 & 0 & 1 & 0 & 0 \\ 0 & 0 & 0 & 0 & 1 & 0 \\ 0 & 0 & 0 & 0 & 0 & 1\end{array}\right] x$

Using accurate $Q$ and $R$ weighting matrices, it produces the control gain:

$$
k=\left[\begin{array}{cccccc}
18.9 & 1.98 & 7.48 & 1.53 & 7.03 & 0.70 \\
-2.22 & 19.4 & -0.450 & 11.9 & -0.770 & 7.03
\end{array}\right]
$$

\subsection{Experimental approach}

In order to study the impact of using the network on closed control loop, the 2-DOF Helicopter control model is built as an NCS. Several nodes are connected through a communication network (Figure 2). The experiments consist of 8 processors. These real-time kernel processors and the network are simulated using TrueTime [11,12]. The network used is a CSMA/AMP (Carrier Sense Multiple Access with Arbitration on Message Priority), it has a rate of $10000000 \mathrm{bits} / \mathrm{sec}$ and not data loss. This protocol is typically used by CAN, minimum frame size of 40 bits, and not data loss. The TrueTime Network block simulates the physical and medium-access layer of various localarea networks. Notice that the network blocks only simulate the medium access (the scheduling), possible collisions, or interference, as well as point-to-point/broadcast transmissions.

Four sensor nodes execute periodic tasks to sense control signals, as well as other additional periodic tasks. Each task has a period $p_{i}$ and time consumption $c_{i}$ (Figure 2). The sensed control signals are $\theta, \psi, \dot{\theta}, \dot{\psi}$. This model has a controller node, depicted on the left side (Figure 2). This controller takes the control law from the FF+LQR module by means of a task, which activates by event. The time consumption of the controller task is the maximum average time it takes to compute the control law. The controller node uses the values from sensors, and sends control outputs $u_{p}$ and $u_{y}$, which correspond to the pitch and yaw voltages. Two actuator nodes, located on the bottom right corner (Figure 2), receive signals from the controller node. Finally, the scheduler node, located on the top right corner (Figure 2), organizes the activity of the other seven nodes, and it is responsible for periodic allocation bandwidth, used by these nodes.

Each node is initialized specifying the number of inputs and outputs of TrueTime kernel block, defining the scheduling policy and creating periodic tasks for the simulation which involves period and consume parameters. The task period defines the time interval between tasks and consume is the task execution time. 
Several Simulink models and Matlab scripts are used to build the model of the helicopter dynamics and they run a simulation of the closedloop response using the position controller. Some tests related to the use of the above scheduling model are presented by Melendez and Benitez [9]. Some faults on the sensors of the helicopter affect the behavior of the system [13]. Figure 3 shows a system's simulation which presents unstable behavior.

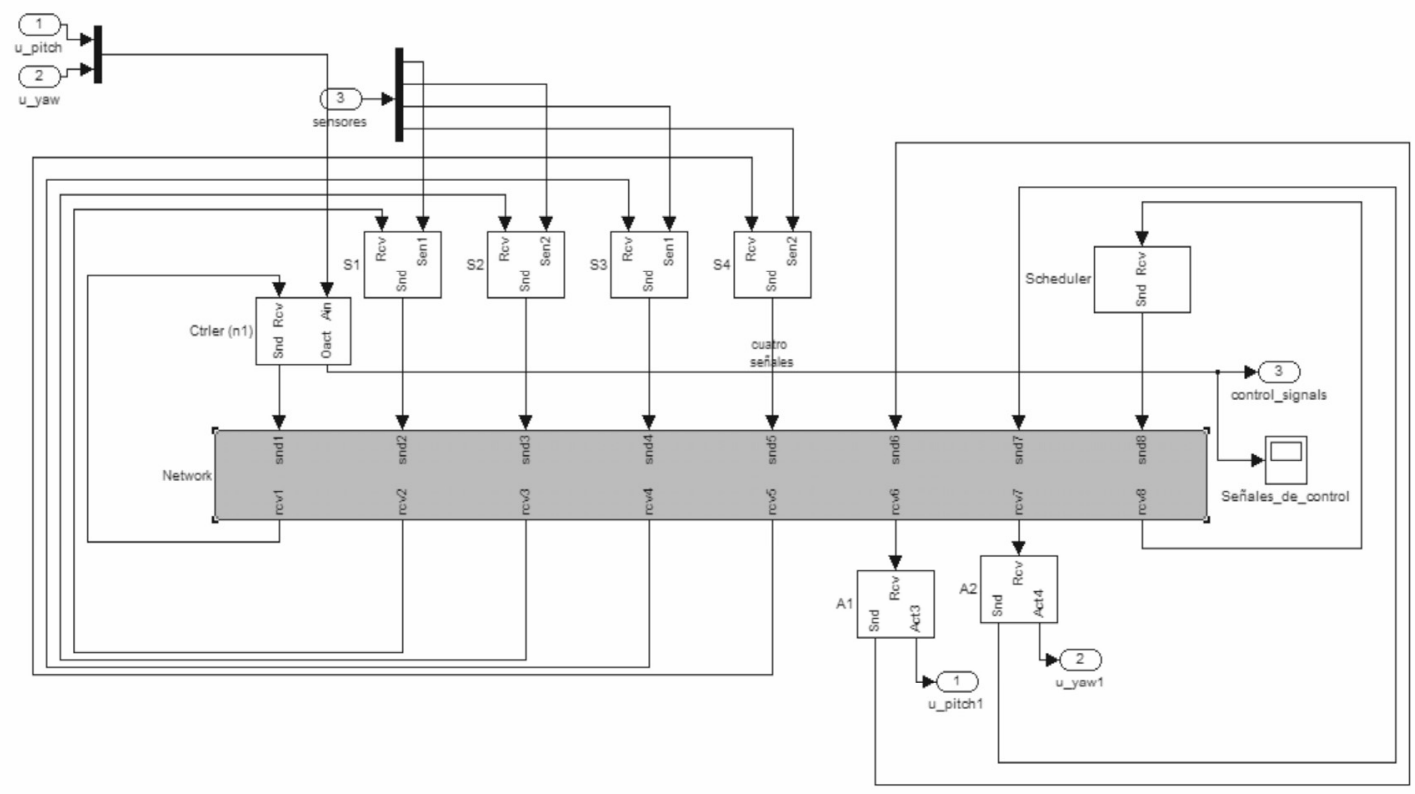

Figure 2. Networked control system in helicopter control model
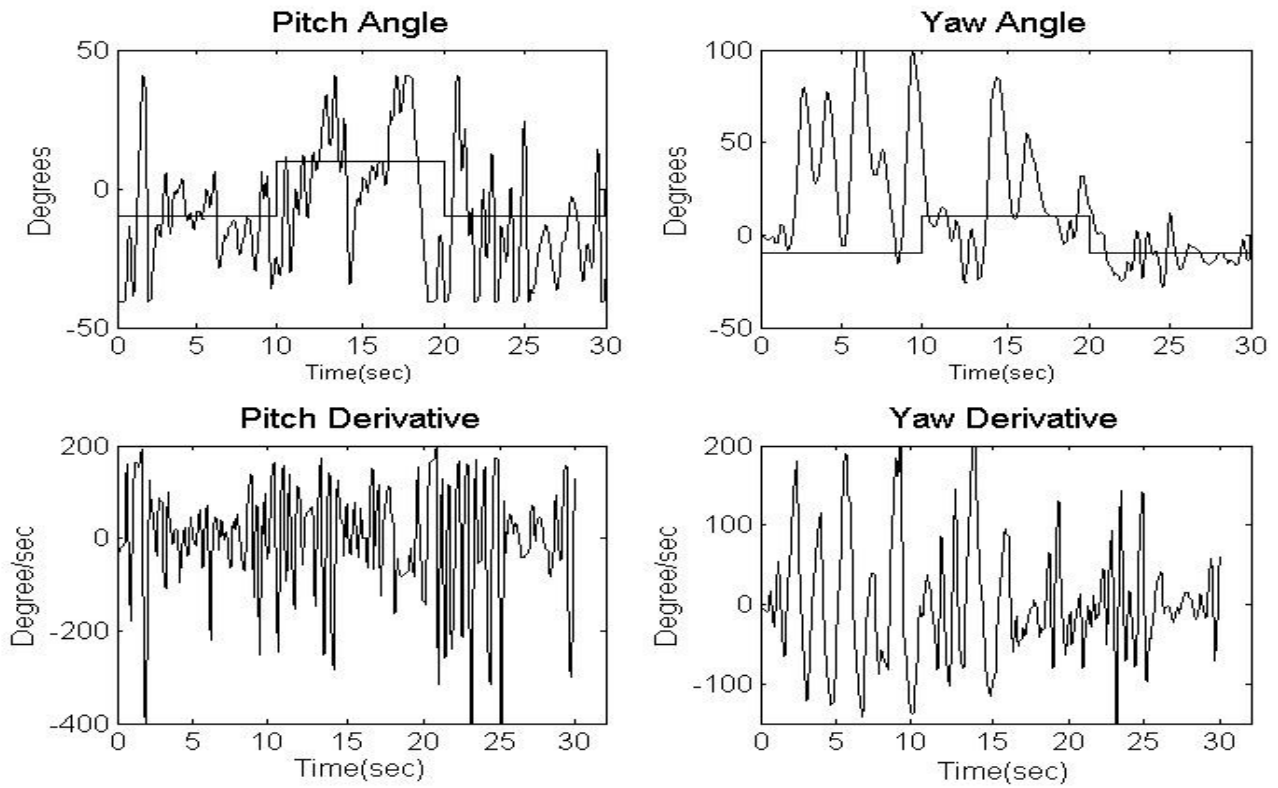

Figure 3. Unstable behavior of the helicopter. 


\section{Frequency transmission scheduling}

An approach to schedule a real-time distributed system based upon modifications on frequency transmission of individual components in the system is presented in [14]; this shows that scheduling of a distributed system can be accomplished through modifications on transmission frequencies into a region where the system performance is not affected. A linear time invariant model in which the coefficients of the state matrix are the relations between the transmission frequencies of each node and through an LQR feedback controller to modify transmission frequencies bounded between maximum and minimum values of transmission. For instance, four measures are very important to implement a closed-loop control cycle in the helicopter system: $\theta, \dot{\theta}, \Psi, \dot{\psi}$ these are sensed by an equal number of nodes which in a distributed manner send sensed data $\theta_{s}, \dot{\theta}_{s} \Psi_{s}, \dot{\psi}_{s}$ to the node controller. Each sensor performs a periodic task with a sampling period $\mathrm{p}_{\mathrm{i}}$.

This approach drives the frequency transmission though three parameters: minimum frequency $f_{m}$, real frequency $f_{r}$ and maximum frequency $f_{x}$. The distributed system dynamics can be modeled as a linear time-invariant system whose state variables are transmission frequencies $\mathrm{f}_{\mathrm{i}}=\frac{1}{\mathrm{p}_{\mathrm{i}}}$ of $n$ nodes involved in it. There is a relationship between node frequencies and external input frequencies which serves as coefficients of the linear system; therefore. it is possible to control the NCS through input vector $u$ such that outputs $y$ are the node frequencies in a nonlinear region $L$ bounded by maximum and minimum transmission frequencies (Figure 4).

The objective of controlling the frequency is to achieve coordination through the convergence of values. Each sensor $i$ has a minimum $f_{m}$ and maximum frequencies $f_{x}$ transmission rates computed offline. The controller node receives these rates through messages from sensors to estimate real transmission frequency $f_{r}$ of the sensors. Let a distributed system with $\mathrm{n}$ nodes or nodes that perform one task $t_{i}$ with period $p_{i}$ and consumption $c_{i}$ each one for $i=1,2, \ldots, n$.

A way to control the dynamic resource scheduling is introducing feedback into dynamic resource management. The basic role of this kind of scheduling is dynamically adjusting the real frequency of transmission. The controlled variables are the real frequencies and the manipulated variable is the sampling period based on minimum frequency.

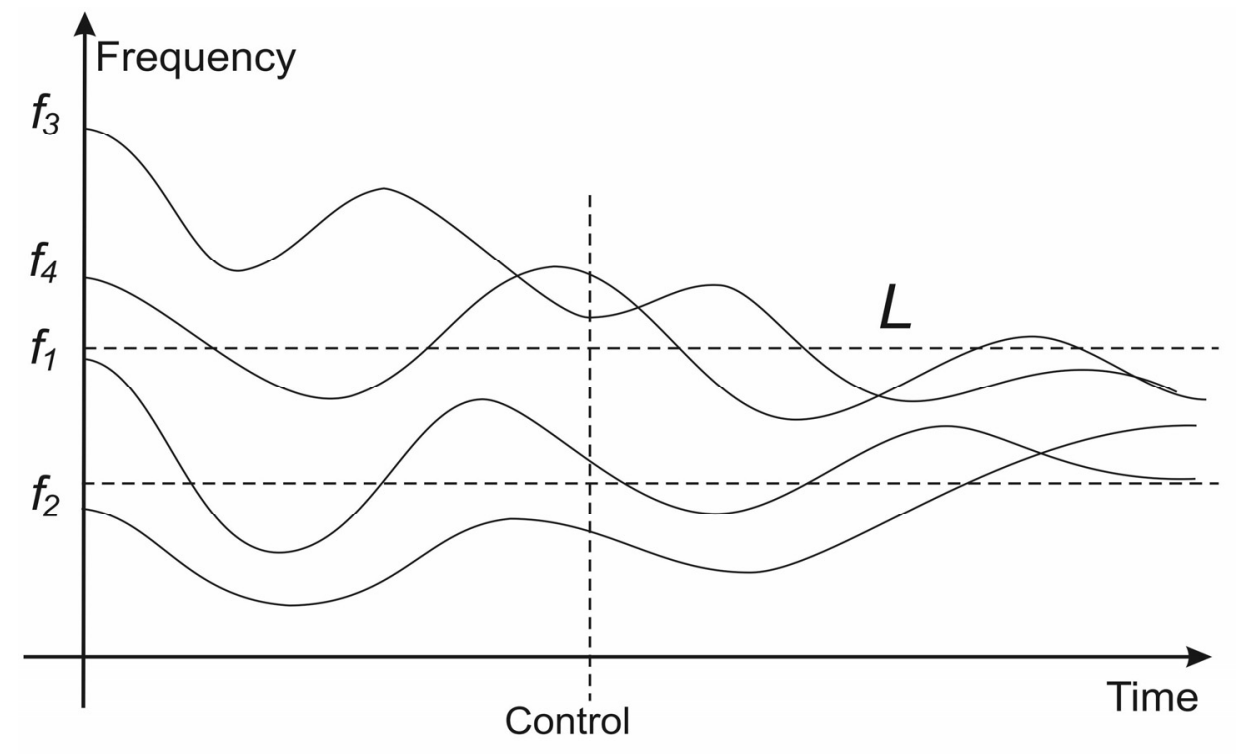

Figure 4. Schedulability region based upon frequency transmission. 
The change in the frequency transmission of each node through the time is represented using the relations of frequencies amongst a particular node and all nodes. It means that the frequency transmission rate of each node is influenced by changes in the transmission rate of other nodes. The frequency transmission dynamics can be modeled as a linear time-invariant system, which state variables $x_{1}, x_{2}, \ldots, x_{n}$ are the real frequencies of $n$ nodes in the network control system. The authors assume that the relationships amongst frequencies $f_{1}, f_{2}, \ldots, f_{n}$ and external input frequencies $\mathrm{u}_{1}, \mathrm{u}_{2}, \ldots, \mathrm{u}_{\mathrm{n}}$ can be computed. The quantity of data sent through the network can be controlled based on modifications of the frequency transmission rate of all nodes. A discrete representation of this system is as follows:

$$
\begin{gathered}
x(k+1)=A x(k)+B u(k) \\
y(k)=C x(k)
\end{gathered}
$$

$A \in R^{n \times n}$ is the matrix of relationships between frequencies of the nodes, $B \in R^{n \times n}$ is the scale frequencies matrix, $C \in R^{n \times n}$ is the matrix with frequencies ordered, $x \in R^{n}$ is a real frequency vector, $y \in R^{n}$ is the vector of output frequencies. The input $u=h(r-x) \in R^{n}$ is a function of reference frequencies and real frequencies of the nodes in the distributed system. Let $a_{i j} \in A$ given by a function of minimal frequencies $f_{m}$ of node $i$ and $b_{i j} \in A$ given by a function of maximal frequencies $f_{x}$ :

$$
\begin{aligned}
& a_{i j}=\varphi\left(f_{m}^{1}, f_{m}^{2}, \ldots, f_{m}^{n}\right) \\
& b_{i j}=v\left(f_{x}^{1}, f_{x}^{2}, \ldots, f_{x}^{n}\right)
\end{aligned}
$$

The control input is given by a function of the minimal frequencies and the real frequencies of node i:

$$
u=h(r-x)=k^{s}\left(f_{m}-f_{r}\right)
$$

$f_{m}$ and $f_{r}$ are the vectors.
Then, the system (13) can be written as:

$$
\begin{gathered}
x(k+1)=A x(k)+B u(k) \\
x(k+1)=A f_{r}(k)+B\left(k^{s}\left(f_{m}(k)-f_{r}(k)\right)\right) \\
\left.x(k+1)=A f_{r}(k)+B k^{s} f_{m}(k)-B k^{s} f_{r}(k)\right) \\
x(k+1)=\left(A-B k^{s}\right) f_{r}(k)+B k^{s} f_{m}(k)
\end{gathered}
$$

Note $k^{s}$ is the control gain defined as the basics of LQR algorithm. Matrices A, B and C are dimensionally correct with the following restriction:

$$
\mathrm{U}=\sum_{\mathrm{i}=1}^{\mathrm{n}} \frac{\mathrm{c}_{\mathrm{i}}}{\mathrm{p}_{\mathrm{i}}} \leq 1
$$

Elements of the matrices for system (13) are defined as follows:

$$
\begin{aligned}
& a_{i j}= \begin{cases}\frac{\lambda\left(f_{m}^{1}, f_{m}^{2}, f_{m}^{3}, f_{m}^{4}\right)}{f_{m}^{j}} & i=j \\
\frac{f_{m}^{j}}{f_{m}^{j}} & i \neq j\end{cases} \\
& b_{i j}= \begin{cases}f_{x}^{i} & i=j \\
0 & i \neq j\end{cases} \\
& c_{i j}= \begin{cases}1 & i=j \\
0 & i \neq j\end{cases}
\end{aligned}
$$

$\bar{\lambda}\left(f_{m}^{1}, f_{m}^{2}, f_{m}^{3}, f_{m}^{4}\right)$ is the greatest common divisor of the minimum frequencies, we will write only $\bar{\lambda}$.

Due to $\quad f_{m}=\left[f_{m}^{1}, f_{m}^{2}, f_{m}^{3}, f_{m}^{4}\right]^{\top}, \quad f_{r}=\left[f_{r}^{1}, f_{r}^{2}, f_{r}^{3}, f_{r}^{4}\right]^{\top}$, $f_{x}=\left[f_{x}^{1}, f_{x}^{2}, f_{x}^{3}, f_{x}^{4}\right]^{\top}$, it is very important to consider the compute time of the task of each node $i$ as an additional state. Using (13), we can rewrite this as follows: 


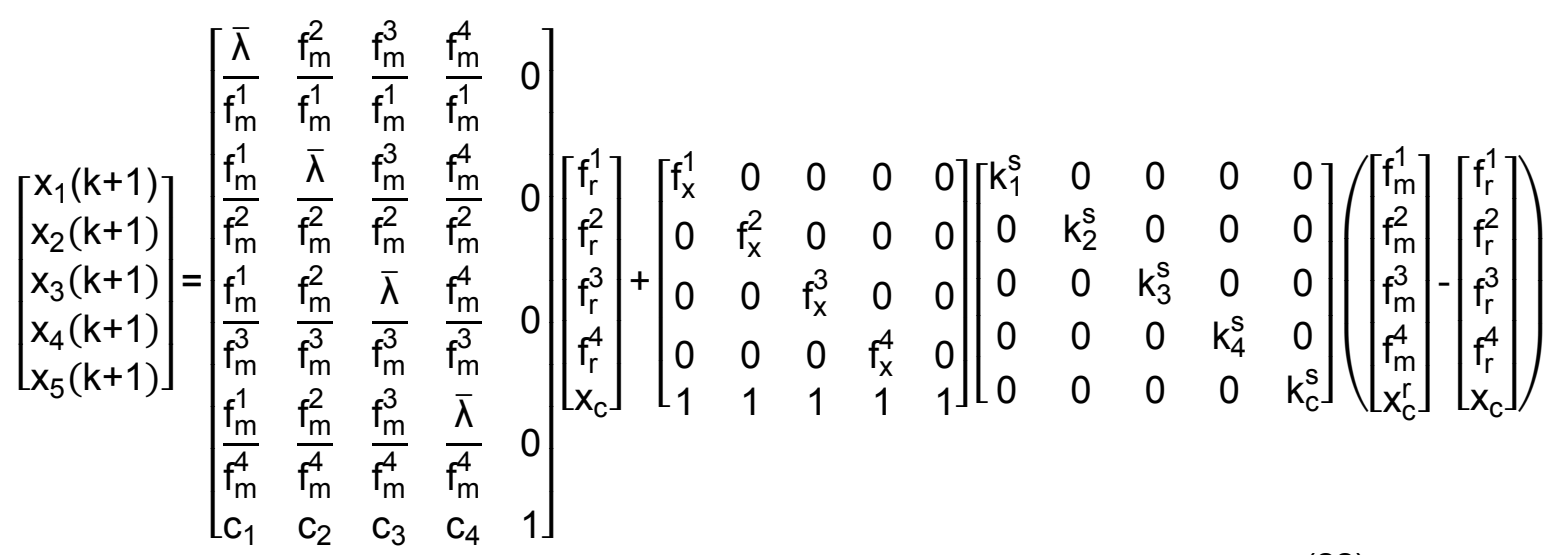

where $c_{1}, c_{2}, c_{3}, c_{4}$ are the consumed times from each process considering periodic tasks within their respective periods and $x_{c}$ are real consume and $x_{c}^{r}$ reference consume.

$$
\left[\begin{array}{l}
y_{1} \\
y_{2} \\
y_{3} \\
y_{4} \\
y_{c}
\end{array}\right]=\left[\begin{array}{lllll}
1 & 0 & 0 & 0 & 0 \\
0 & 1 & 0 & 0 & 0 \\
0 & 0 & 1 & 0 & 0 \\
0 & 0 & 0 & 1 & 0 \\
0 & 0 & 0 & 0 & 1
\end{array}\right]\left[\begin{array}{l}
f_{r}^{1} \\
f_{r}^{2} \\
f_{r}^{3} \\
f_{r}^{4} \\
x_{c}
\end{array}\right]
$$

thus,

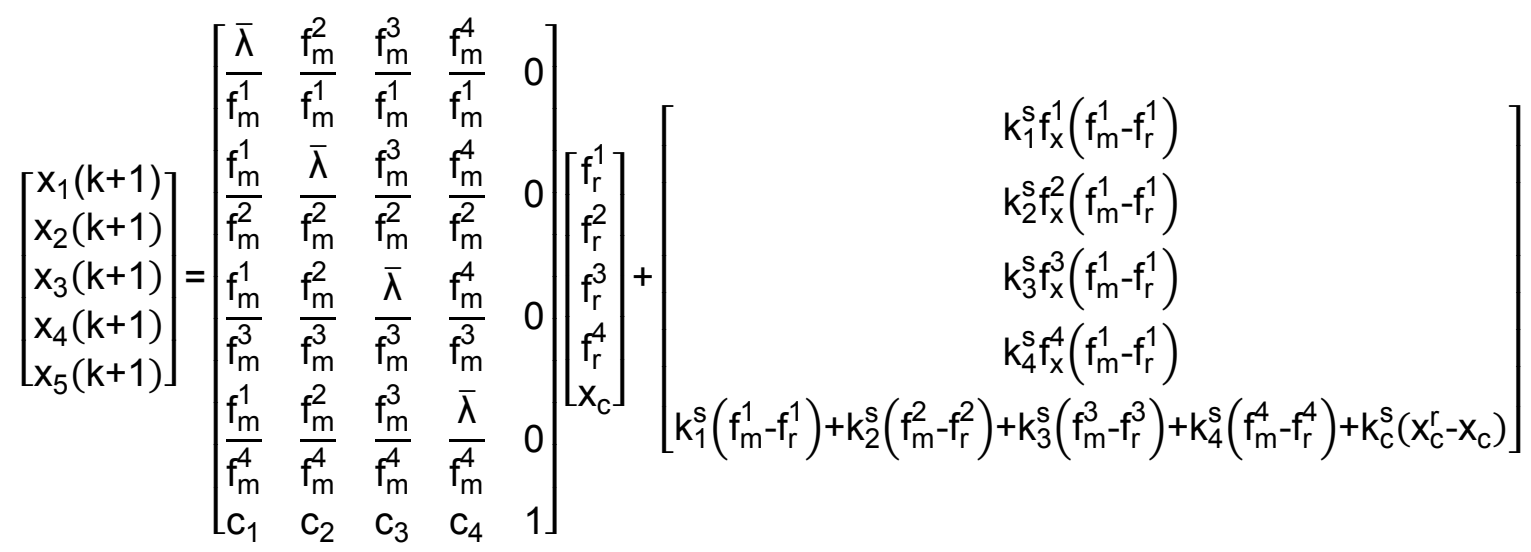

$$
\left[\begin{array}{l}
y_{1} \\
y_{2} \\
y_{3} \\
y_{4} \\
y_{c}
\end{array}\right]=\left[\begin{array}{lllll}
1 & 0 & 0 & 0 & 0 \\
0 & 1 & 0 & 0 & 0 \\
0 & 0 & 1 & 0 & 0 \\
0 & 0 & 0 & 1 & 0 \\
0 & 0 & 0 & 0 & 1
\end{array}\right]\left[\begin{array}{c}
f_{r}^{1} \\
f_{r}^{2} \\
f_{r}^{3} \\
f_{r}^{4} \\
x_{c}
\end{array}\right]
$$


Since the data transmission frequency of the network nodes using an NCS can be high because of uncertainties during sensing, the system may become unstable, similarly low data frequency transmission results in undersampling which goes to control performance weakness. Therefore, it is important to manage the sensor nodes dynamically to ensure somehow that they are restricted to a schedulability region where the system is stable.

Figure 5 shows data transmission from the sensor to the scheduler sending frequency data through the network. The scheduler uses frequency information to compute the frequency transmission algorithm.

An effective data interchange between nodes feeds the frequency transmission model which modifies the node frequency, thus the network utilization is proper.

\section{Numerical simulations}

From this implementation, several results are presented in terms of different transmission frequency values (sampling periods) of the sensor nodes and their modifications using the frequency transition model implemented on scheduler node. How the system responds to the frequency transmission strategy is presented in the following graphics showing the evolution of the control signals during a time interval of $60 \mathrm{~s}$.

Large sampling periods result in insufficient data to compute the control law in the controller node, this degrades quality of control. Nevertheless, small sampling periods increase network bandwidth demand. Figure 6 shows a typical fault scenario where some transmission frequency values of the sensor nodes are outside of the schedulability region where the system could experiment undersamplig or overload. A criterion to evalute control quality performance is IAE (Integral of absolute error). It means, the sum of absolute value of the difference between reference $r(t)$ and output $y(t)$ signals during time. The value of IAE obtained using arbitrary values of the frequency (Table 2) was 682 in pitch angle signal and 208 to yaw angle signal.

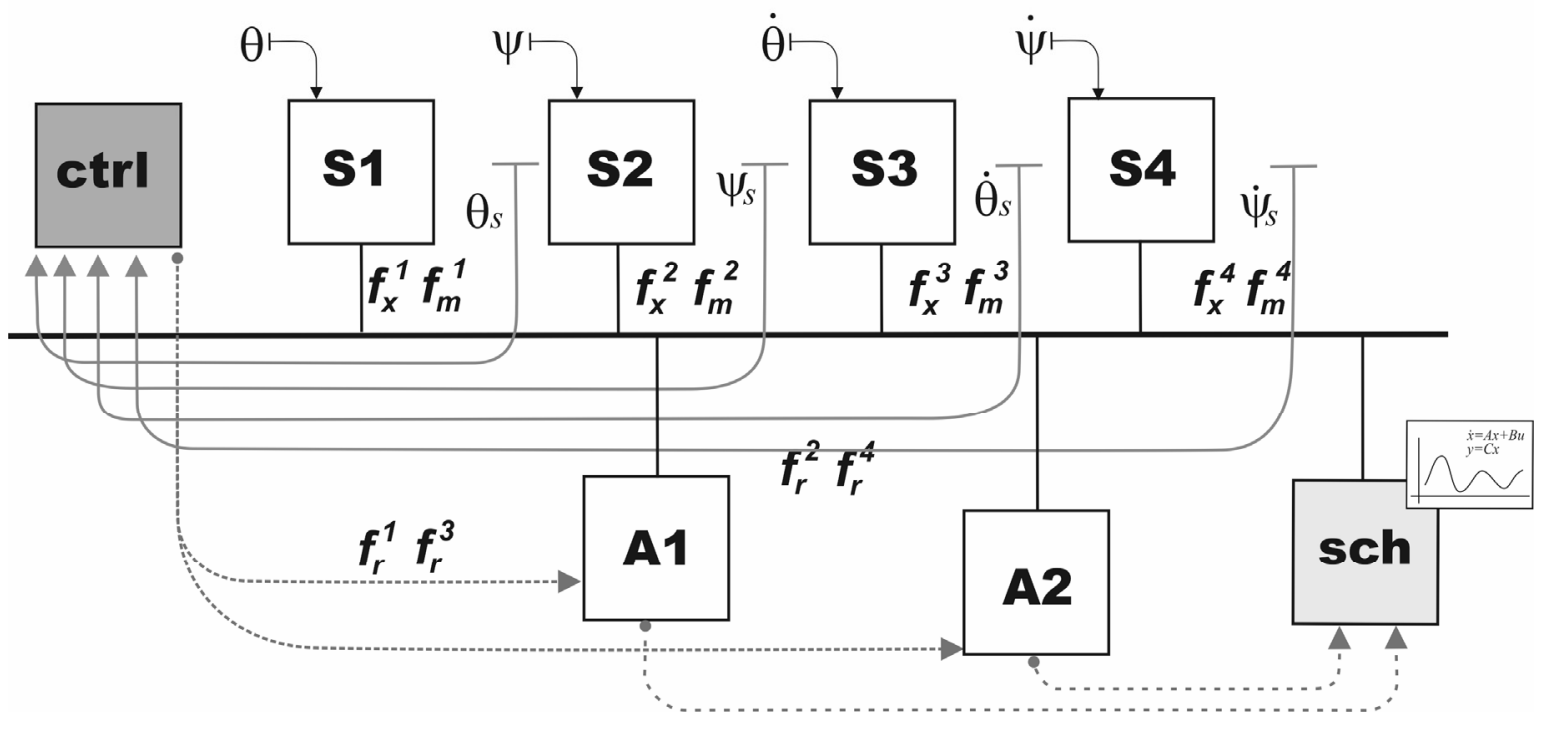

Figure 5. Communication between nodes from the sensors to the scheduler. 

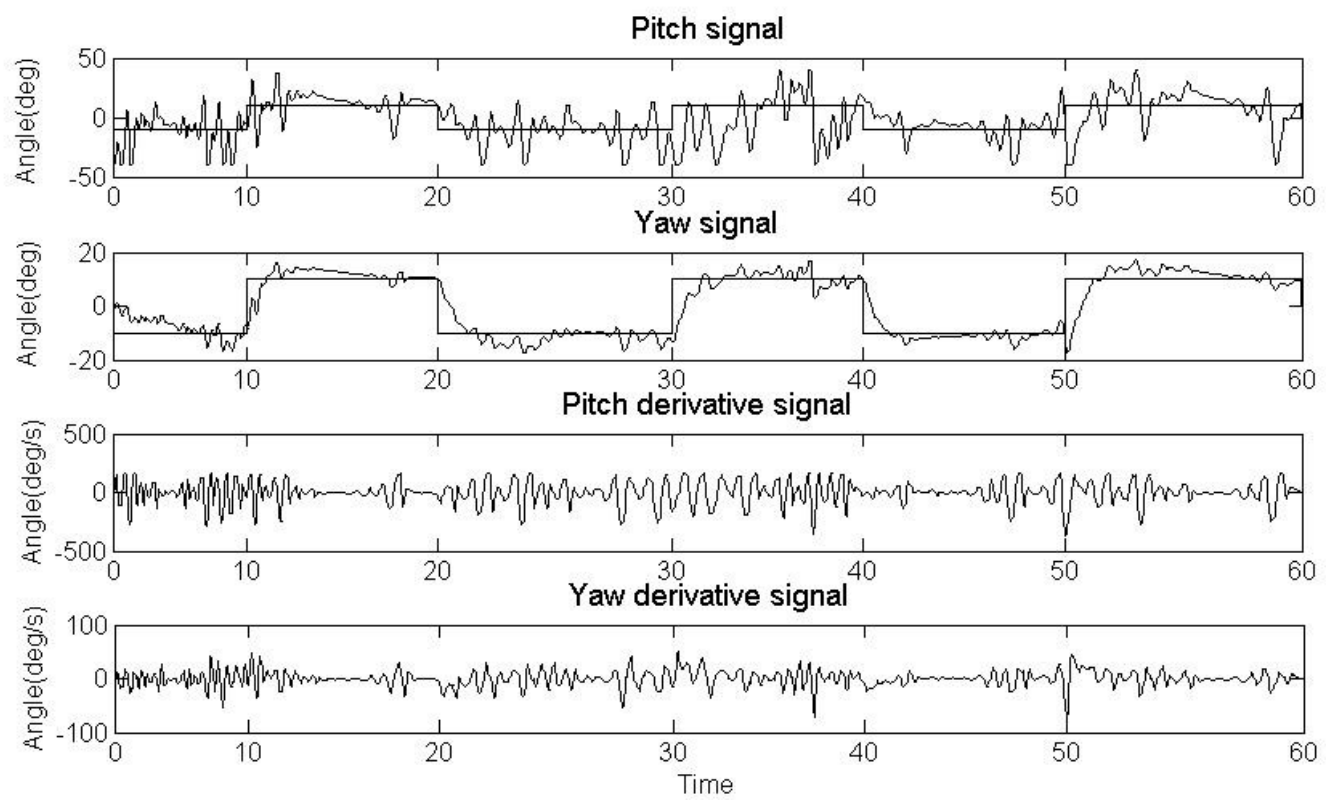

Figure 6. Typical fault scenario. Transmission frequency values are outside of schedulability region.

\begin{tabular}{|c|c|c|}
\hline Node & $\begin{array}{c}\text { Frequency } \\
(\mathrm{Hz})\end{array}$ & Period \\
\hline S1 & 90 & 0.011 \\
\hline S2 & 59 & 0.0175 \\
\hline S3 & 23 & 0.043 \\
\hline S4 & 48 & 0.0215 \\
\hline
\end{tabular}

Table 2. Arbitrary frequencies and respective sampling periods.

Figure 7 shows different values of the IAE of pitch angle signal and yaw angle signal using sampling periods between 1 millisecond and 20 milliseconds, it means $1000 \mathrm{~Hz}$ and $50 \mathrm{~Hz}$.
Figure 8 shows the behavior of the system using the frequency transition model. Nominal frequencies start outside of the schedulability region (frequency set $F_{0}$ ), the system transmits at this rate for 15 seconds. In second 15 , the scheduler node changes the transmission frequency values (frequency set $F_{1}$ ) based on control of frequencies; in time interval 15-30 s., the system is stable; in second 30 , a new change is induced with frequency values outside the schedulability region (frequency set $F_{2}$ ), the system becomes unstable again. Finally in second 45, the frequency transition model changes the frequencies (frequency set $F_{3}$ ). 

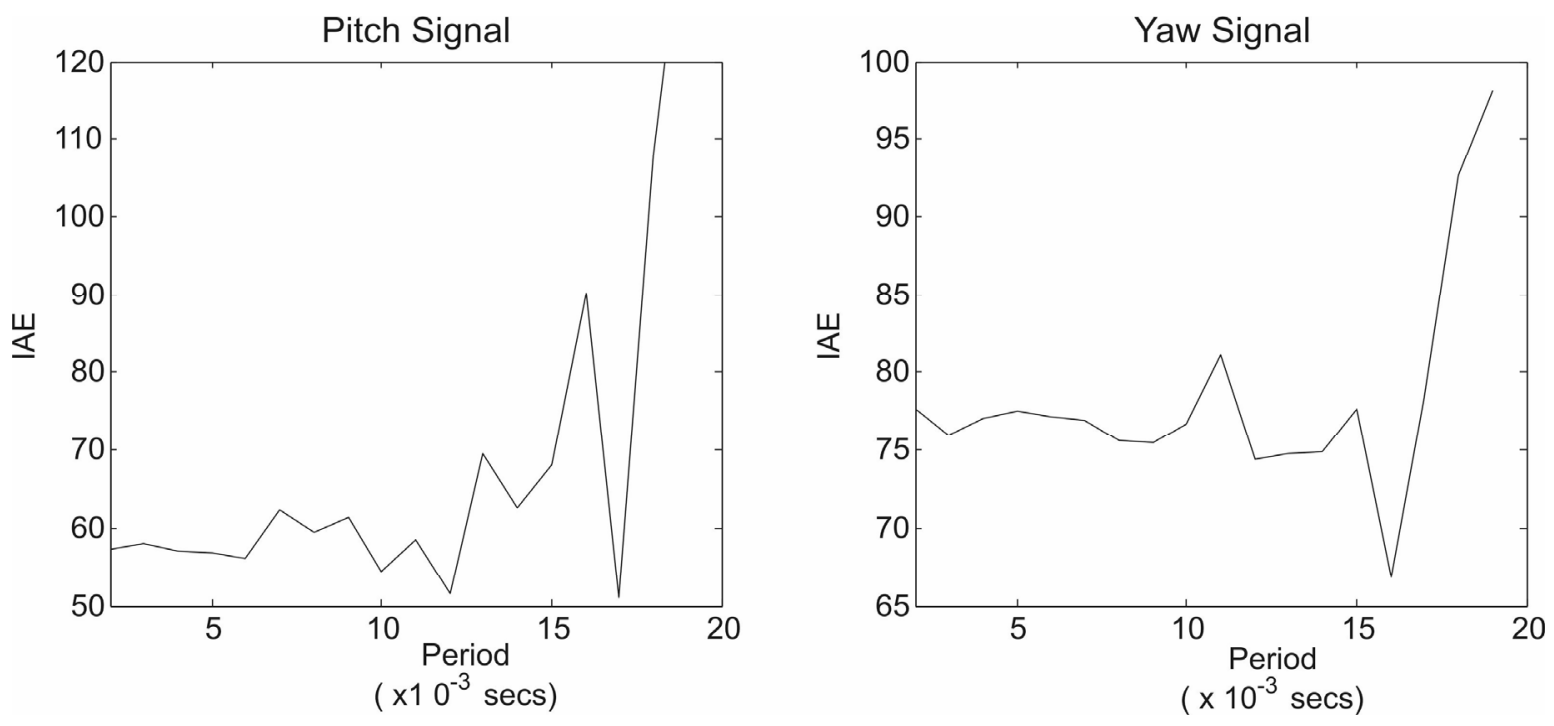

Figure 7. Pitch angle and yaw angle signals and their respective values of IAE for several sampling periods.

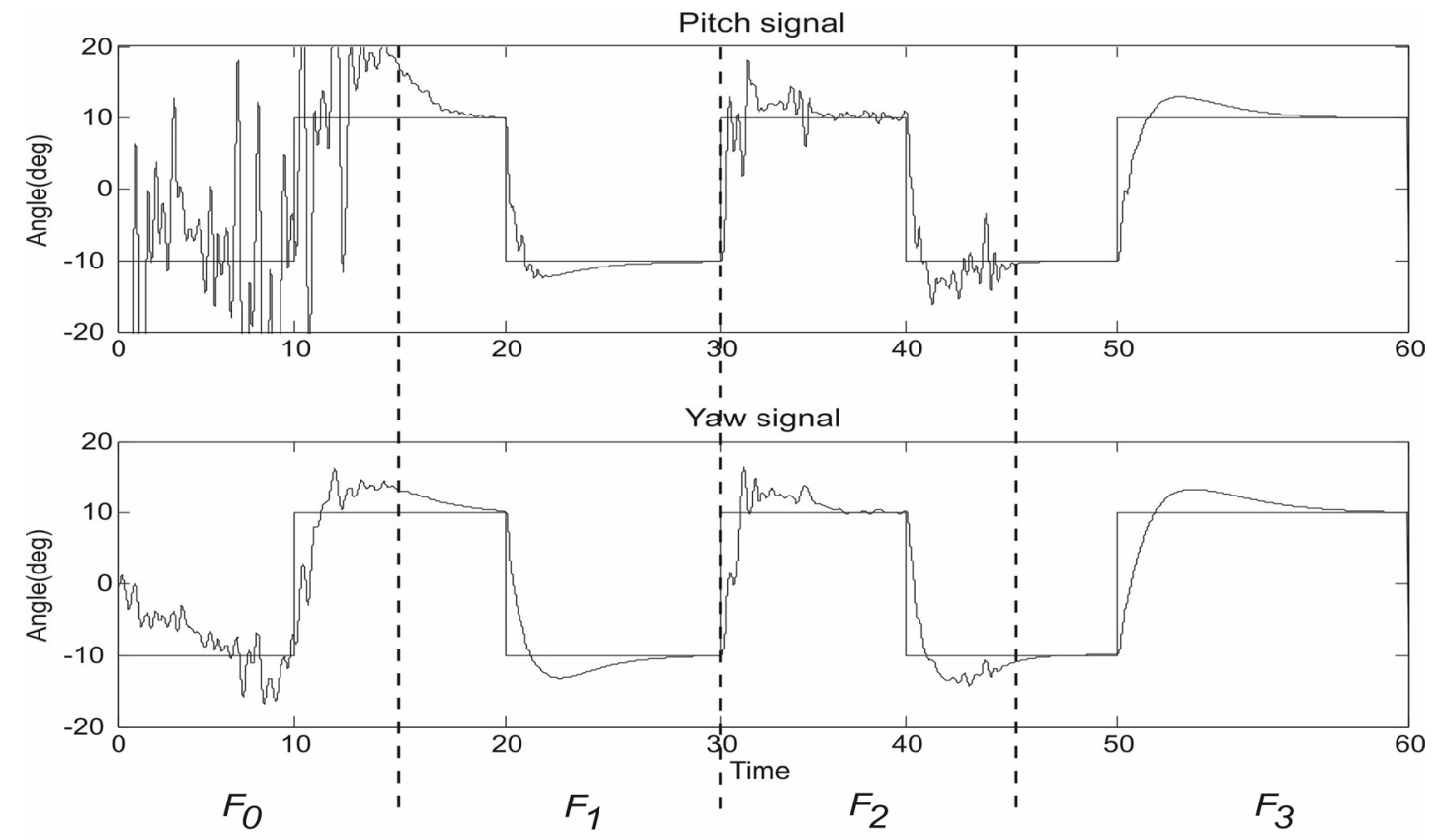

Figure 8. Changes on transmission frequency impact system behavior.

Third nominal frequency, set F2, contains constant values outside the schedulability region. 
The integral of absolute error in this scenario results in 240 to pitch signal and 160 to yaw signal. Table 3 shows the frequencies used at each interval of 15 seconds to each sensor node.

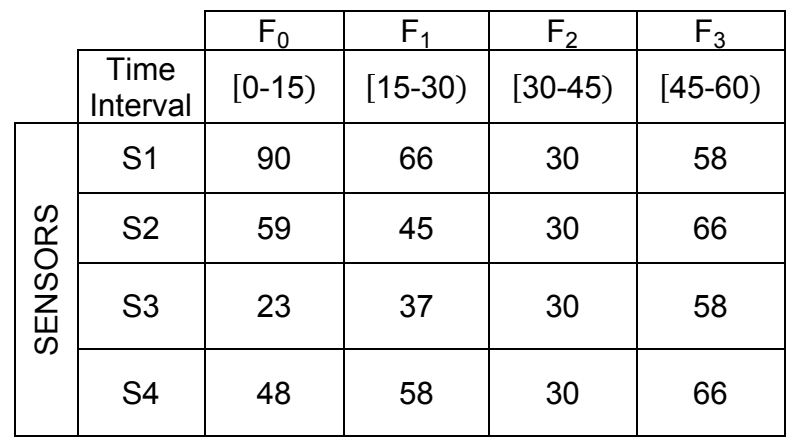

Table 3. Changes of frequencies each 15 seconds.

Finally, Figure 9 shows the response of the system when noise is induced after the first frequency change. The effectiveness of the frequency transition model is slightly lower than in the previous scenario but it works to correct instability. Nominal frequencies start outside of the schedulability region (frequency set $F_{0}$ ), the system transmits at this rate for $15 \mathrm{~s}$. In second 15 , the scheduler node changes transmission frequency values (frequency set $F_{1}$ ), based on control of frequencies; in time interval $15-30 \mathrm{~s}$, the system is stable; in second 30 , a new change is induced with frequency values outside the schedulability region (frequency set $F_{2}$ ), the system becomes unstable again. Finally, in second 45, the frequency transition model changes frequencies (frequency set $F_{3}$ ).

The integral of absolute error results in 161 for pitch signal and 460 to yaw signal. Table 4 shows the frequencies used at each interval of 15 seconds to each sensor node.

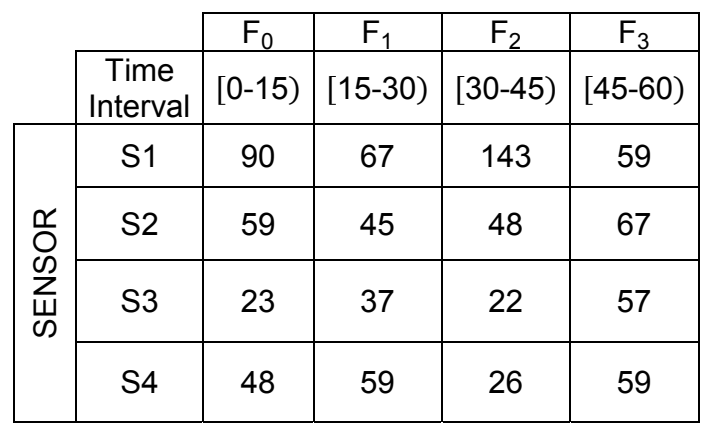

Table 4. Changes of frequencies each 15 seconds.

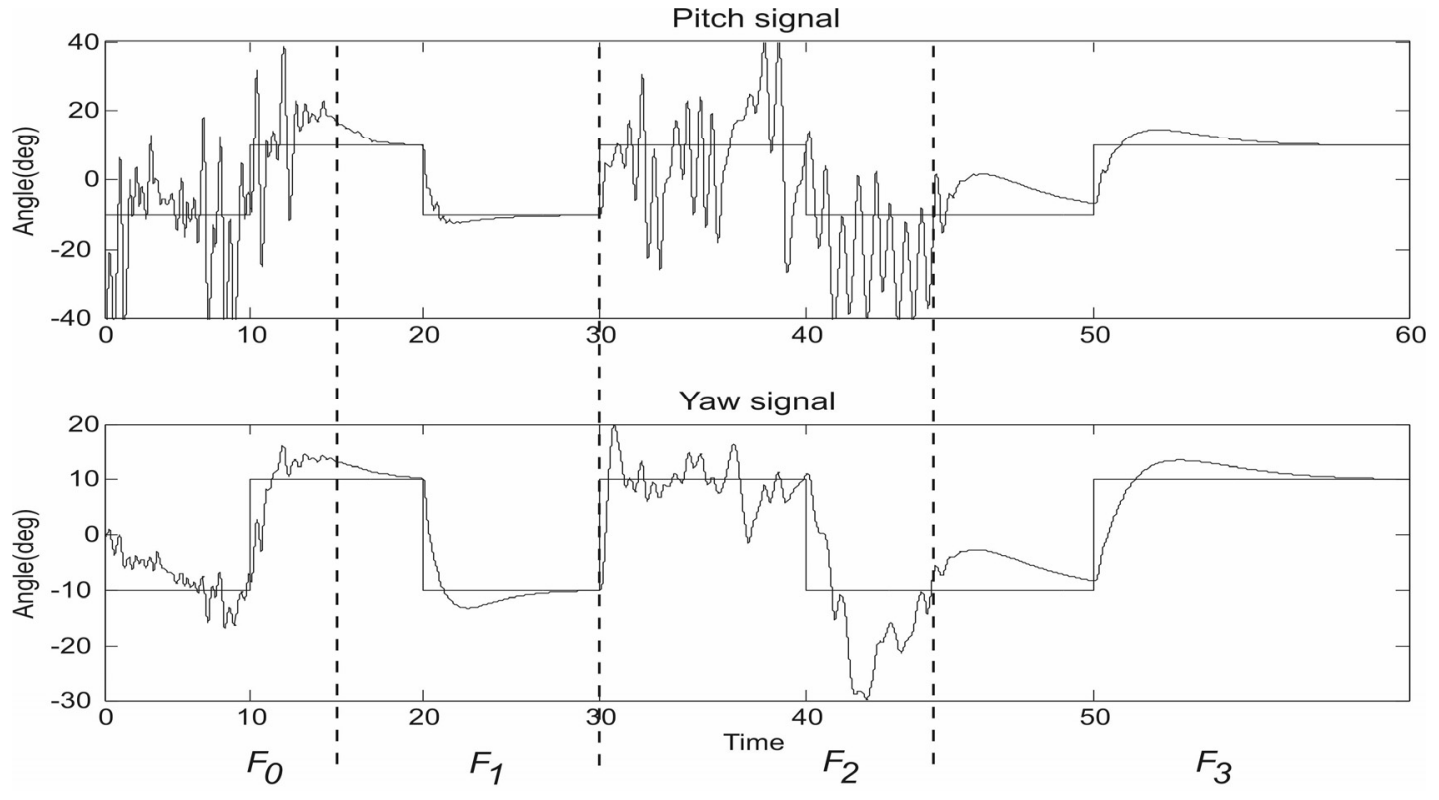

Figure 9. Changes on transmission frequency impact system behavior. Third nominal frequency set, F2, contains variable values outside the schedulability region. 


\section{Conclusions and further work}

In this work, the authors propose a scheduling approach to particularly access to the network of an NCS. As a case of study, a prototype of a helicopter was used. In this prototype, a distributed system that performs flight control functions was included to show the scheduling approach performance. Scheduling strategies are mainly based on modification of sampling periods and, therefore, the frequency of data transmission. In this paper the authors show some results of numerical simulations using a model for managing data transmission frequencies in a distributed system, particularly an NCS; based on an LQR control scheme that dynamically balances the amount of data generated by sensors, this in order to avoid the amount of data sent through the network being greater than those possible to be transmitted; it tries to avoid network congestion and eliminate long waiting periods. This approach dynamically adjusts frequencies of data considering the participation of several nodes in a distributed system, however, it is also a centralized proposal because the frequency transmission dynamical system is calculated only by the scheduler.

An interesting area for future work is to consider that each node could compute the control transmission frequency and reach a consensus from all involved nodes ( nodes' system) to manage globally the access to the network, thus, to obtain a distributed scheduling. The exploration of the effect of increasing the network load to calculate an accurate frequency transmission would be the following work to be developed.

\section{Acknowledgements}

The financial support from grants PAPIIT-UNAM 103310, ICYTDF PICCO 10-53, and CONACYT (PhD grant) are especially appreciated.

\section{References}

[1] Halevy, Y., Ray A.,. Integrated Communication and Control Systems: Part I-Analysis, Journal of Dynamic Systems, Measurement, and Control, Vol. 110, 1988, pp. 367-373

[2] Lian, F., Moyne, J. Tilbury, D.,. Time delay modeling and sample time selection for networked control systems, Proceedings of ASME-DSC, Vol. XX, 2001, New York, USA.

[3] Lian, F., Moyne, J. Tilbury, D., Network design considerations for distributed networked for distributed control systems, IEEE Transactions on Control Systems Technology, Vol. 10, No. 2, 2002, pp. 297-307.

[4] Yu, M., Wong, L., Chu, T., Xie, G.,.Stabilization of Networked Control Systems with Data Packet Dropout and Network Delays Via Schitching System Approach, Proceedings 43rd IEEE conference on decision and control, Atlantis, Paradise Island, Bahamas, 2004, pp.14-17.

[5] Lian, F., Moyne, Yook, J., Otanez, P., Tilbury, D., Moyne, J.,. Design of sampling and transmission rates for achieving control and communication performance in networked multi-agent system, Proceedings of American Control Conference, Denver, USA, Jun. 4-6, 2003, pp. 3329-3334.

[6] Lian, F., Moyne, J., Tilbury, D.,.Network architecture and communication modules for guaranteeing acceptable control and communication performance for networked multi-agent systems, IEEE Transactions on Industrial Informatics, Vol. 2, No. 1, 2006, pp. 12-24.

[7] Branicky, M., Liberatore, V., Phillips, S., Networked control system co-simulation for co-design, Proceedings of American Control Conference, Denver, USA, June 46, 2003, pp. 3341-3346.

[8] Peng, C., Yue, D., Gu, Z., Xia F., Sampling period scheduling of networked control systems with multiplecontrol loops, Mathematics and Computers in Simulation, VoL. 79, 2009, pp. 1502-1511.

[9] Menéndez, A., Benítez-Pérez, H., An interaction amongst real time distributed systems performance \& global scheduling, Journal of Applied Research and Technology, Vol. 8, August 2010, pp. 177-185.

[10] Quanser:2-DOF Helicopter, User and Control Manual. Quanser, Speciallity Experiments:2-DOF Helicopter, 2006. 
[11] Ohlin, M., Henriksson, D., Cervin, A., TrueTime 1.5 Reference Manual, Department of Automatic Control, Lund University, 2007.

[12] Cervin A., Henriksson D., Lincoln, B., Eker, J., Arzen, K., How does control timing affect performance? Control Systems Magazine, Vol. 23, Num. 3, 2003, pp. 16-30.

[13] Heredia, G., Ollero, A., Bejar, M., Mahtani, R., Sensor and actuator fault detection in small autonomous helicopters. Mechatronics, Vol. 8, 2008, pp. 90-99.

[14] Esquivel-Flores, O., Benítez-Pérez, H., Méndez, E., Menéndez, A. 2010. Frequency Transition for scheduling management using dynamic system approximation for a kind of NCS, ICIC Express Letters, part B: Applications, Vol. 1, Num. 1, pp. 93-98.

[15] Benítez-Pérez, H., García-Nocetti, F., Reconfigurable Distributed Control, Springer, 2005. 